\title{
Chronic Postoperative Endophthalmitis Caused by Actinomyces meyeri
}

\author{
Vasileios G. Peponis Spyridon E. Chalkiadakis \\ Efstratios A. Parikakis Panagiotis G. Mitropoulos \\ 'Ophthalmiatreio' Athens Eye Hospital, Second Eye Clinic, Athens, Greece
}

\section{Key Words}

Actinomyces meyeri - Cataract surgery · Chronic endophthalmitis · Pars plana vitrectomy . Topical antibiotics

\begin{abstract}
We report a female patient who developed chronic endophthalmitis after an uneventful cataract surgery. Cultures of aqueous humor and a vitreous sample showed positivity for Actinomyces meyeri. Intense anterior segment inflammation and a less evident impairment of the patient's posterior segment led us to treat her vigorously with pars plana vitrectomy combined with intraocular and topical antibiotics. The patient achieved a good recovery of vision without the need to remove the intraocular lens and to add systemic drugs. To the best of our knowledge, this is the first reported case of an intraocular infection caused by $A$. meyeri after small-incision clear corneal phacoemulsification.
\end{abstract}

\section{Case Report}

A 65-year-old Caucasian woman was admitted to our hospital for a planned cataract extraction in her right eye (OD). Preoperative examination revealed a visual acuity of $20 / 50$ s.c. OD and the presence of a nuclear sclerotic cataract grade 3 . The patient's personal and family medical history was unremarkable. The operation was uneventful and the adjusted phacoemulsification time was $0.294 \mathrm{~min}$. One milligram $/ 0.1 \mathrm{ml}$ intracameral cefuroxime was injected at the end of the operation, and the patient was put on topical gentamicin and $0.1 \%$ dexamethasone drops q.i.d. Postoperative visual acuity was 20/25 s.c. OD.

Two weeks later, and while the patient was still taking the prescribed drops, she was examined in our emergency department complaining of a sudden vision reduction in her operated eye. In fact, her visual acuity was reduced to $20 / 60$ s.c. OD. On clinical examination, the patient did not report any pain, and adnexa were normal. Slit-lamp examination revealed a corneal edema with Descemet's folds. The anterior chamber was deep, with mild Tyndall effect, +2 cells and no signs of hypopyon. Intraocular pressure was $16 \mathrm{~mm} \mathrm{Hg}$. After dilation of the pupil, an apparent biofilm was covering the anterior surface of the intraocular lens which did not obscure fundoscopy, and in the capsular bag an evident fibrinous exudate could be found. The vitreous was normal, with no cells and signs of vitritis. These

Vasileios G. Peponis, MD, MSc, PhD 'Ophthalmiatreio' Athens Eye Hospital, Second Eye Clinic 
findings resulted in a good retinal view without any signs of abnormality. The patient was therefore admitted for an emergency 23-gauge pars plana vitrectomy. The activity in the vitreous was not markedly elevated, and after a vitreous sample had been taken and the vitreous removed, a posterior capsulotomy was made using a vitreous cutter. Meticulous rinse of the capsular bag was performed, and the trocars were removed after the administration of $1 \mathrm{mg} / 0.1 \mathrm{ml}$ vancomycin and amikacin to the vitreous cavity.

After the operation, the patient was advised to use internally fabricated topical fixed-combination fortified vancomycin-amikacin q.i.d. as previously described [1], combined with topical drops of $0.1 \%$ dexamethasone suspension q.i.d. After a 1-week microbiological investigation, cultures revealed positivity for Actinomyces only, and in particular for Actinomyces meyeri. To match the microbiological results, the treatment was modified with the use of topical drops of ofloxacin and steroids q.i.d. The patient tolerated this treatment well and her visual acuity began to recuperate. At present, she is under constant follow-up and her best-corrected visual acuity is currently $20 / 40$ c.c. with $+0.50 \mathrm{DS} /+1.50 \mathrm{DC} \times$ $065^{\circ}$, with no improvement noticed after pinhole insertion.

\section{Discussion}

Actinomycosis is an infection caused by the filamentous, Gram-positive, non-acid-fast, non-spore-forming bacteria Actinomyces spp. They belong to the order of Actinomycetales, family Actinomycetaceae, and genus Actinomyces.

The first report available reporting ocular infection due to a species of Actinomyces comes from Roussel et al. [2]. They reported a case series of postoperative endophthalmitis for which different species of Actinomyces were incriminated, but not A. meyeri. Nonetheless, this study demonstrates various differences from our case report. First of all, the report was made during the extracapsular cataract extraction era, whereas in our report the patient developed endophthalmitis after a small-incision clear corneal cataract extraction. This is a factor of major safety against the ab externo entrance of microorganisms into the ocular microenvironment. Moreover, the pharmacological approach was different between these two studies; in our report, technological and pharmacological research has permitted a better control of the intraocular infection and thus better final results. In addition to this, the surgical intervention was less traumatic, and the intraocular lens was not removed, which did not impair the patient's recovery. Finally, to the best of our knowledge, this is the first time A. meyeri is isolated from a site of infectious endophthalmitis as the only suspect for its occurrence. Our hypothesis is that the microorganism's low virulence, combined with the prompt, decisive and evidencebased response, resulted in the excellent clinical outcome (fig. 1 , fig. 2 , fig. 3 ).

In cases of chronic postoperative infectious endophthalmitis, the recommendation of the European Society of Cataract and Refractive Surgeons (ESCRS) guidelines [3, 4] consists of topical and systemic antibiotic treatment. If no resolution occurs, recommendations revert to pars plana vitrectomy with capsulectomy and intraocular lens removal.

In our case, the inflammatory response seemed quite clearly restricted to the anterior segment, with only mild vitreous opacification, corresponding to grade 1 cellular infiltration in the vitreous. Therefore, we opted to not remove the intraocular lens, and after the capsulectomy we irrigated the capsular bag with balanced salt solution containing no antibiotics. 


\begin{tabular}{c|l|l|l}
$\begin{array}{c}\text { Case Reports in } \\
\text { Ophthalmology }\end{array}$ & $\begin{array}{l}\text { Case Rep Ophthalmol 2011;2:95-98 } \\
\text { DOI: 10.1159/000326062 }\end{array}$ & $\begin{array}{l}\text { Published online: } \\
\text { March 5, 2011 }\end{array}$ & $\begin{array}{l}\text { O 2011 S. Karger AG, Basel } \\
\text { ISSN 1663-2699 } \\
\text { www.karger.com/cop }\end{array}$ \\
\hline
\end{tabular}

In conclusion, we report a case of chronic infectious endophthalmitis where, for the first time, A. meyeri has been clearly found to be the causative agent. The therapeutic regimen included a pars plana vitrectomy without the removal of the intraocular lens, a capsulectomy, the irrigation of the capsular bag with balanced salt solution only and the injection of intraocular antibiotics. Finally, we prescribed topical antibiotics and antiinflammatory drops without the need to hospitalize the patient and without the prescription of systemic antibiotics. The clinical result was very positive for the patient.

\section{Disclosure Statement}

The authors have no financial or proprietary interest in the materials presented herein.

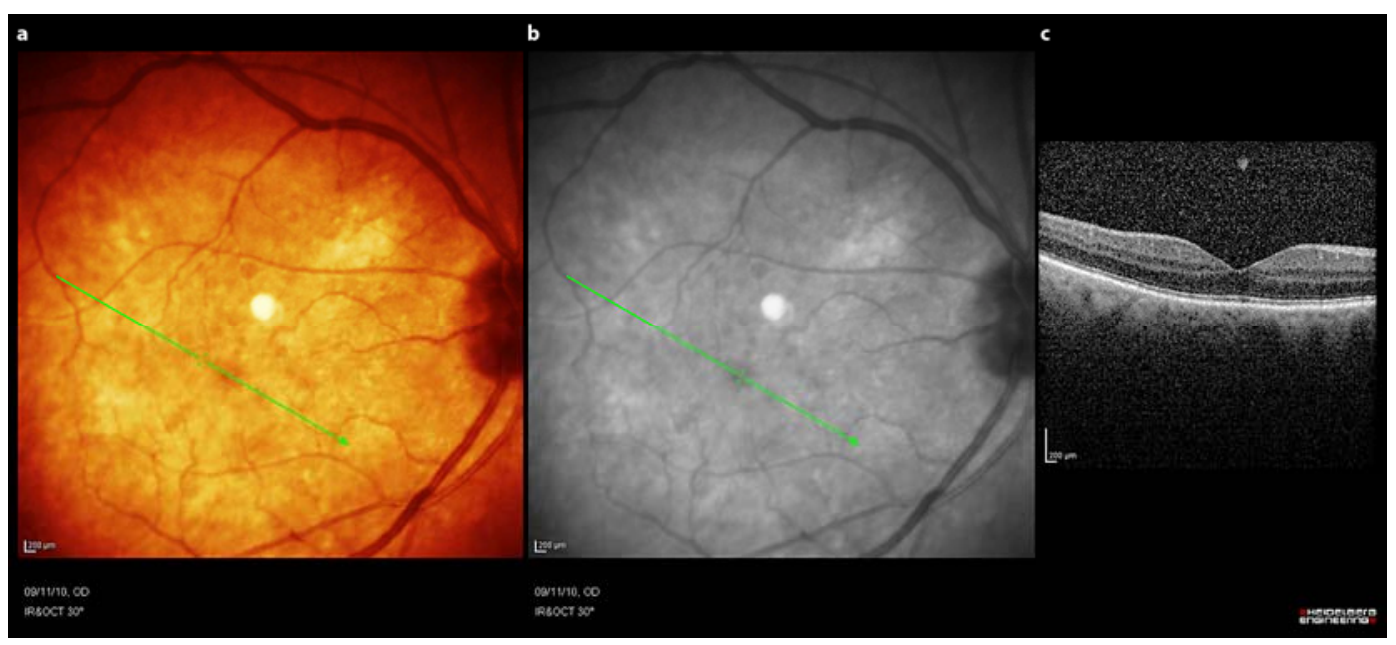

Fig. 1. Color (a) as well as black and white (b) scanning laser ophthalmoscopic images of the fundus. c Cross-sectional optical coherence tomography (Spectralis HRA) of the fundus. 


\begin{tabular}{l|l|l|l} 
Case Reports in & $\begin{array}{l}\text { Case Rep Ophthalmol 2011;2:95-98 } \\
\text { DOI: 10.1159/000326062 }\end{array}$ & $\begin{array}{l}\text { Published online: } \\
\text { March 5, 2011 }\end{array}$ & $\begin{array}{l}\text { ○ 2011 S. Karger AG, Basel } \\
\text { ISSN 1663-2699 } \\
\text { www.karger.com/cop }\end{array}$ \\
\hline
\end{tabular}

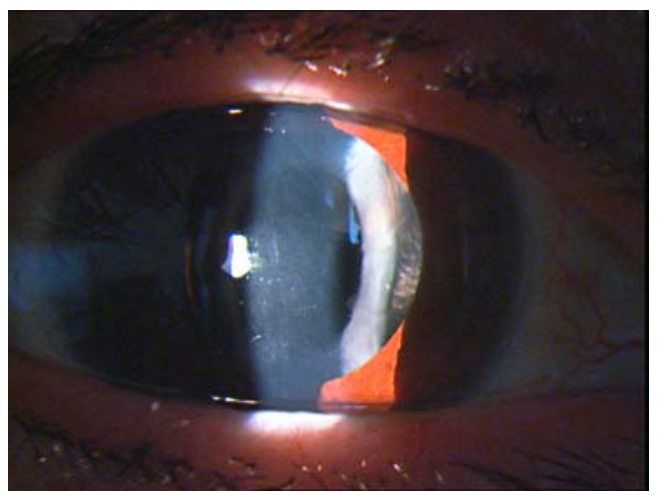

Fig. 2. Postoperative image of the eye showing only a mild corneal edema.

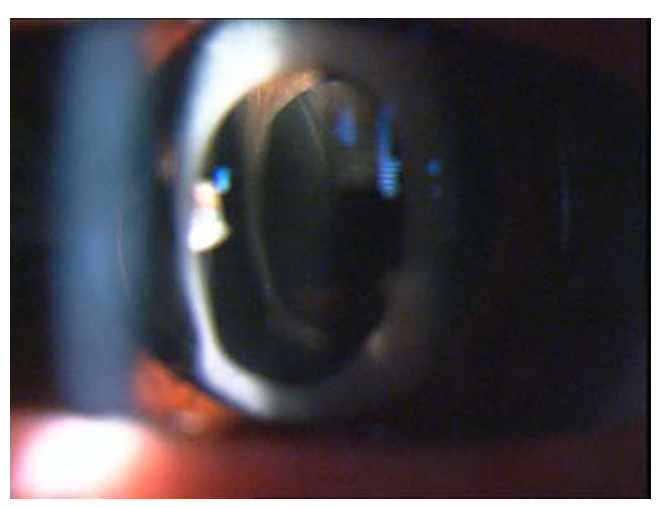

Fig. 3. Postoperative image of the eye showing a clear capsular bag and the intraocular lens in situ.

\section{References}

1 Chiang CC, Lin JM, Chen WL, Chiu YT, Tsai YY: Comparison of topical fixed-combination fortified vancomycin-amikacin (VA solution) to conventional separate therapy in the treatment of bacterial corneal ulcer. Eye (Lond) 2009;23:294-298.

$\longrightarrow 2$ Roussel TJ, Olson ER, Rice T, Meisler D, Hall G, Miller D: Chronic postoperative endophthalmitis associated with Actinomyces species. Arch Ophthalmol 1991;109:60-62.

- 3 Endophthalmitis Study Group, European Society of Cataract \& Refractive Surgeons: Prophylaxis of postoperative endophthalmitis following cataract surgery: results of the ESCRS multicenter study and identification of risk factors. J Cataract Refract Surg 2007;33:978-988.

4 Results of the Endophthalmitis Vitrectomy Study. A randomized trial of immediate vitrectomy and of intravenous antibiotics for the treatment of postoperative bacterial endophthalmitis. Endophthalmitis Vitrectomy Study Group. Arch Ophthalmol 1995;113:1479-1496. 